\title{
Vision Logic and Aesthetic Evolution
}

\author{
Feng Zhu \\ School of Art and Design, Nanchang Hangkong University, Nanchang 330063, China \\ E-mail: szfszfszf@hotmail.com
}

\begin{abstract}
Visual logic is the most basic logic principle that human beings' aesthetic taste is based on. Through conducting search on aesthetic taste, we found out the common rule for aesthetic evolution, which is also an evolution process in a sense of meaning. Firstly, visual system apperceives aesthetic objects in a mode similar to computer searches. In the process of apperception, visual system is controlled by "collective unconsciousness" standard formed through the deposition of "collective" experience and by "individual unconsciousness" formed through the accumulation of "individual" experience. The brain clears up the messages through visual logic, and makes deduction, after which the brain could obtain accurate visual apperception. Under proper circumstances, aesthetic resonance is generated. Further, the aesthetic taste of the group could be sublimed, which in turn exerts impact on the "unconsciousness" aesthetic standard of individuals and collective groups. This is the basic way for the evolution of aesthetic taste and is the evolution logic which occurs momently.
\end{abstract}

Keywords: Visual logic, Aesthetic taste, Art logic

Mainly, the researches on visual logic are conducted for the sake of psychology and biology. Parts of physics and informatics are covered too. This paper is to illustrate the formation and evolution of aesthetic rules in the field of shaping art, which does not simply explain the evolution modes of aesthetic evaluation but aims at pointing out that artistic works creation should undertake the functional role to direct and promote aesthetic evolution.

Firstly, gratitude shall be given to the works of Francis Crick, The Astonishing Hypothesis. After reading this book, the contents of this paper were overthrown and revised when the composing of this paper was nearly completed. The experiments on visual illusion in large quantity described in session one of the book convince us that the saying "seeing is believing", which is commonly known as a truth, is so unreliable, which happens to be one of the main logic backgrounds and sources that this paper originally want to conduct researches on. After adjustment, the research is conducted on the basis of the following understandings on vision:

Apperception is not the true first hand material. Visual messages go through a large quantity of parallel processing which are related to nerve cells and are transferred to and are sensed by the brain apperception systems in a mode similar to "the winner is the king". Here parallel refers to that a large number of nerve cells work simultaneously rather than process information one by one in serial. Only by this way can it be guaranteed that all visual objects are "seen" at instantaneous state. The way that the apperception system senses the information is to sense simultaneously with different intensity. For example, when you are collecting information in a short time from pictures spread on the floor, the main elements that will influence your evaluation include "your concern", the position of the pictures, the number of similar pictures, memory, searching rules, and simple logic organization and detail filling necessary for making evaluation. Many pictures may not be explicitly sensed by you because of the aforesaid reasons. For apperception system, such circumstance will result in the situation where a large account of information is seen but is not sensed. Because the final evaluation must be completed by logic organization and detail filling, there is the possibility for incorrect apperception. A typical example is that visual apperception system takes initiative to fill the blind spots. That is to say that in apperception process, the subjective consciousness is not involved in behaviors, which is a process of true unconsciousness and is extremely similar to the processing mode of computer searching. To put it in another way, in this process, it is easy for inertial processing and falsity to occur, which controls visual logic thinking and further influences aesthetic taste.

Visual logic refers to the causal relationship and corresponding rules followed in the process of visual apperception. To make analysis on it and to make deduction, certain visual principles could be obtained, researches based on which exert great influence on shaping art and even visual aesthetic taste. Generally speaking, the sameness rate of aesthetic evaluation standards is determined by the similarity rate of visual experiences of the evaluation-makers, which covers geographical features and cultural background. At present, the popular view is that usually, different aesthetic main bodies tend to be the same in value direction and are slightly different from each other in details. It is obvious that such saying is not supported by sufficient evidence. Or we could say that such saying exists in practice and application process as a self-convinced point. 
It is shown in a large number of researches that aesthetic taste standards of different regions, different peoples and different ages are not completely consistent with each other. The same as that many self-convinced concepts are finally easily overthrown by practices, the otherness of aesthetic taste is larger than we have imagined. From the perspective of vision, we could not recognize the decoration ways of peoples in remote areas. Even, we rebel at their tastes. Similarly, sometimes, the images that we do not appreciate are popular in other countries. There is a hypothesis question, which is if the otherness of visual experience on certain images is large enough, is it possible that their evaluation standards are completely different from or even are opposite to each other. Tim Burton applied a melody to describe the elimination of Martian (Note 1), which is at least understandable in art logic, which is to say that if this kind of otherness exists, what should be done is not to improve but to avoid. We believe that aesthetic taste is not transcendent and does not remain the same.

Then, how is aesthetic taste is generated?

As a corresponding mood generated by apperception, is aesthetic taste generated by unconscious instinct or does it follow certain causal rules? The research on visual logic well answers this question. It is composed of the following points:

\section{The influence of prediction and hint on aesthetic instinct}

Aesthetic sense is a kind of adapting degree. When the development of an issue complies with the thinking of the observers or with the prediction of the observers, the comfort sense of corresponding extent will generate. F.L.Pogson describes the sense of beauty in his brochure on time extension as follows, "The reason why hurried and discontinuous movements are not beautiful is that each of such movement is self-sufficient and they do not predict their following movements. The reason why curve is more beautiful than broken lines is that the curve changes its direction momently and each new direction is predicted by its previous direction" (Pogson, 2004). The prediction and hint in vision brings easy and joyous sense to aesthetic main bodies. Curve predicts certain track. Each dot on the line gives hint to the generation of the next dot, which complies with the thinking of observers, is easy, and could be controlled so as to generate the sense of beauty. The hints among dots on broken lines are uncertain. The appearance of many unpredictable direction conflicts with the thinking of observers and causes discomfort and nauseous feelings.

It could be seen that even the aesthetic instinct of original stage contains causal rules and follows certain visual logic rules. "The said instinct refers to rational experience. It puts us into the internal part of an object so as to comply with unique and inexpressible stuff of the object" (Pogson, 2004). The generation of original aesthetic taste is through the prediction capacity of aesthetic main body and the hints given by the objects. Connection and relation of the two are established so that different reactions in psychology and body are triggered. Artistic works make use of this kind of relations and apply clearing up, pruning, fixing and piling up to enhance the function of hints to realize the growth of the extent of sense of beauty.

\section{Then influence of "collective unconsciousness" on aesthetic taste}

The reason why usually people tend to be the same in aesthetic values is that human beings have similar civilian background and the aesthetic taste of various visions and touch and even the spirits accumulate to form a relationship where aesthetic body and its living circumstances mutually depend on each other, become accustomed to each other and recognize each other, which is a natural result of evolution. Because its contents could be found in the hearts of "all people" and have common characteristics, it is called "collective unconsciousness". The original aesthetic taste obtained from inheritance has certain regional and time characters. Due to long term deposition, within specific period, it controls aesthetic taste to the maximum and limits aesthetic evolution

As "collective group" of aesthetic main body, it could be less than "all people" and also could be more than "all people". In daily life, it is easy to understand the "collective unconsciousness" less than "all people". For example, the original aesthetic tastes of different peoples, phylum, regions, and systems have certain differences. To understand more than "all people", we need to take into account all conscious main bodies. For example, whether animals have aesthetic taste, if yes, to what extent is it consistent with human beings? Compared to human beings, it is usually believed that animals do not have aesthetic deduction capacity. However, we must admit that animals have certain visual taste and good image memory capacity. Because they live under similar environment to human beings, in many detailed aesthetic evaluation, they could form a wider "collective group" with human beings. "Collective unconsciousness" and "individual unconsciousness" influence "unconscious" aesthetic taste together. It needs to be supplemented that from the angle of art logic, usually, any item could become the main body of certain type if only they have different adapting and tastes.

\section{The wakening and memory of visual remaining image}

From the perspective of psychology, aesthetic taste containing "individual unconsciousness" is represented in the accumulation and deposition of aesthetic individual's vision and psychological experience. A typical case is the paraphrase of Oedipus's complex made by Freud. However, in real life, such complex is not so obvious. There is a 
more common and easier explanation, which is that the memory and wakening of visual remaining images have special connotation to aesthetic taste and control the corresponding aesthetic evaluation of individuals.

A metaphor for it is that usually it is easy for us to accept items with which we are familiar. There are two kinds of demonstrations for the said familiarity. The first is the memory of the known images. For example, a grown up will be deeply attracted by the images which are similar to the images that they cognized in their childhood. You may find that the opposite sex that you are obsessed with is familiar to you in certain angle, parts or gaseity. It could be used to explain the complex of missing hometowns. The same circumstance also occurs in our endurance extent on ugly items. Usually, we will find that due to the passing of time, some extremely ugly images will not be so difficult to endure after some periods. Even, we become infatuated with them.

The second is the wakening of visual remaining images. Many aesthetic images are more complicated in cognition than intuition. Usually, the wakening of visual remaining images could only be realized under certain proper circumstances and the wakening process requires the participation of logic deduction. We could say that the memory of visual remaining images and its wakening function on logic deduction accelerates aesthetic evolution. The aesthetic taste on abstract works are also based on this type of memory and logic wakening, which makes aesthetic evaluation on new works of famous painters easier than the works of unfamous works.

\section{Aesthetic taste deduction, empathize, resonance and aesthetic sublimation}

In the following part, we will analyze the aesthetic activities that we participate consciously. Firstly, let us analyze the empathize phenomenon in aesthetic taste. Different from the depth psychology, empathize in aesthetic taste only occurs on the observers (the aesthetic evaluation main bodies). However, it is recommended that creators of modern arts should take into account this aspect and apply it. It could belong to subjective empathize in creation. There are two types of empathizes of observers and there is one type of empathize in the creation of artistic works. Now, let us explain in detail the occurrence of the three types of empathizes.

The observers' feelings are transferred to the works, which usually occurs in movies and television series. For example, the audiences could easily think themselves as the default images when the movie or TV works are broadcasting. He (she) might imagine himself or herself to be she or he or he (she) is being hurt (or is committing crime). However, the function of such empathize narration far exceeds the function of visual logic. We determine to explain it by visual logic in visual aesthetic taste range. When we are appreciating a painting, we will unconsciously transfer our feelings to the objects of the painting. What is interesting is that such transfer does not only occur on human objects. It will make corresponding transformation according to the change of the painting's subject. The priority level order of such transformation is characters (including main characters, peoples, genders, ages, and body shapes), animals (their sizes, their positions on the painting and their relationship with human beings), plants, architectures (or ships, planes, and other man made objects. when many pieces appear at the same time, please refer to animal level), mountains and rocks (or sceneries that have detailed shapes in the vicinity) and distance sceneries. It could also occur in series sceneries that the feelings are transferred to a group of objects. When we appreciate the water fowls painted by Badashanren (Note 2), we will feel strong loneliness, which is particularly obvious when appreciating eastern painting artistic works, which is the aesthetic empathize, "audiences are the objects".

In western paintings, due to the influence of human thoughts, the artistic works usually adopt the third person visual observing method. There is no painter in the picture but the painter is also everywhere. The painter gazes at the contents of the painting at all times. Therefore, the aesthetic empathize occurs between observers and painters, which means "audiences are painters". For example, when we gaze at the large sized painting works of Anselm Kiefer (Note 3), we usually will feel inexplicable sense of inhibition. This kind of inhibition is absolutely not to empathize to certain wall of post but is brought by the entire dark climate. The one who truly feels this kind of climate is not others but the painter. Let us take a buoyant example. The empathize on Mona Lisa is not to transfer the feelings of Mona Lisa to the observers but inherits the feelings of Leonardo da Vinci and a typical attitude is the third person mood of "falling in love with Mona Lisa" (however, to tell the truth, to the author, the charm of Mona Lisa is not so big as advertised by the western media, or this might be caused by the difference between westerners and easterners in "collective unconsciousness"). It could be said that the artist transfer his or her feelings on the painted main body to the audiences and combine the audiences' feelings, and form the individual aesthetic taste on works.

For artistic logic, artistic creation process will also integrate creation empathize similar to Tongjia. Strictly speaking, such circumstance is not true feeling transfer. It is shown at artistic creation and through engrafting method and it enables the image of the painted main body exceeds its inherit image character and further "gives" this kind of feelings to the audiences. For vision, easy methods, such as to make slight change in partial shaping and bright and dark combining position, could bestow a drape newspaper with the quality sense of steel or cotton cloth. This kind of empathize usually is used to express the internal mood of the works and is the common method in artistic creation. The same handling is used in personification more. What is more, this kind of empathize is also applied in other artistic 
creation fields, for example, "Troubled by the times-flowers bring tears; Dreading parting-birds startle the soul"(Note 4). The flowers and birds are bestowed with the feelings of human beings by way of empathize.

Similar to empathize, the resonance of artistic aesthetic taste also occurs in the aesthetic field where consciousness involves. As an important mark for the acceptance and entrance of artistic vision into climax stage, resonance follows closely after the occurrence of empathize and is the important moment for observers to release moods. At this stage, visual apperception system basically stops working. The brain is under artistic logic thinking working period. We say that at that time, the brain is working at high speed, ceaselessly analyzes the existing messages, deducts unknown rules (and imagine), and sends to the body various instructions (have various features, such as being cold or hot in hands and feet, flourishing, being dry in lips and being incoherent). At the same time, resonance could also be shown in feeling reaction among painters, aesthetic main bodies and various audiences. When individual feelings return to "collective feelings", it is usually accompanied by the generation of aesthetic sublimation. After disposition, the aesthetic sublimation will generate new round of aesthetic common recognition. It will transform into "collective unconsciousness" of aesthetic taste and change radically the existing aesthetic standards and further realize the aesthetic evolution.

With the increase of intensity, resonance and aesthetic sublimation process will last for considerably long time, just as we usually say "circulating around the girder for three days" (Note 5). The common appreciating background, resonance and sublimation will draw nearer the evaluation of various aesthetic groups. The gradually frequent art exchanges of the world indicate the tendency of united aesthetic standards of the participating groups.

\section{Aesthetic deduction-the requirement of logic evaluation on works creation}

In the process of visual art creation, another point needs our attention, which is the requirement of logic evaluation on works creation. In another article, the author made the following analysis on the basic requirement on artistic creation. The basic requirement of artistic creation is to expand knowledge on aesthetic taste of human beings on the basis of existing aesthetic taste and to guide the responsibilities of evolution. To put it into other words, as the aim of basic researches, artistic creation (note: it is not the artistic creation for application) (Note 6) is to constantly make innovations. Similarly, the said artistic innovation does not mean that the artists talk to themselves. This kind of innovation must be constructed on the basis of visual logic and must be consistent with aesthetic common recognition ("unconscious" aesthetic taste). It uses the sensitivity (Note 7) formed through long term accumulation "individual unconsciousness" aesthetic taste) to find the truth different from experiences. When certain amount of unknown messages are added into visual apperception system, the artists must search for rational explanations through artistic logic analysis and practical methods. Here, it is mostly shown by the tie-in of unknown messages and the existing original aesthetic tastes (this is also the hardest stage of artistic innovation. Artists need to make lots of practices. Like the tie-in of two lines, they not only should do researches on unknown messages, but also should do researches and adjust the original "unconsciousness" aesthetic taste. It is explained in the previous part that no matter what kind of "unconsciousness" it is, individual or collective group, it is not that there is no rule to follow. For a mature artist, a majority of people who do not receive art education could fully understand and skillfully apply it. The extended use of this noun is to explain the needs of hierarchy, which is the core point of this paper). Once the possibility of tie-in is found, artists will be on the wires. Here, it could be explained as the finding of artistic inspirational sense or sudden realization, which is a concept in artistic logic. In the operation process of tie-in, consciousness needs to conduct a new round of message conclusion, sorting and setting up searching rules, which are done by the brains. The subjective consciousness just sends out the concluded instructions. Because the tie-in of the new and old messages could not be completed without logic organizations, usually it needs consciousness to fill in. Therefore, subjective consciousness needs to resolve the filling in at this stage. It is reflected in external behaviors as the artistic expression and artistic creation process that we are yearning for. For the new visual aesthetic taste of improved artistic works creation, it raises aesthetic quality.

When the aforementioned works are presented to common audiences, it complies with the supreme principle of artistic creation, which is "comply with but beyond our sense". Under the guiding of artistic works, the audiences upgrade their aesthetic tastes through artistic logic thinking. Hence, when recognizing the works, the audiences also recognize their self-accepting and understanding capacity. Because the audiences have explicit sense on the upgrading of their aesthetic character, they could have academic resonance with creators, which is the value of the artistic creations.

Therefore, we obtain a full picture for the process of aesthetic evolution: visual system senses the aesthetic objects in a way similar to computer searching. In the process of apperception, visual system is controlled by "collective unconsciousness" standards formed through the deposition of "collective" experiences and by "individual unconsciousness" formed through the accumulation of "individual" experiences. The brain clears up the messages through visual logic, and makes deduction, after which the brain could obtain accurate visual apperception. Under proper circumstances, the aesthetic resonance is generated. Further, the aesthetic tastes of the group could be sublimed, which in turn exerts impact on the "unconscious" aesthetic standard of individuals and collective groups. This is the 
basic way for the evolution of aesthetic taste and is an evolution logic which occurs momently. No matter it is "consciousness" or "unconsciousness", they both comply with the logic rules of the nature and is the inevitable result of natural evolution.

\section{References}

Benjamin, Walter. (2002). The Work of Art in the Age of Mechanical Reproduction. Beijing : China City Press.

Baldwin, Elaine. ect.(2004). Introducing Cultural Studies. Beijing : Higher Education Press.

Crick Francis. (2007). The Astonishing Hypothesis. Changsha : Hunan Science and Technology Press.

Dong, Xiaoying. (1997). Narration Artistic Logic Introduction. Beijing : Social Science Literarture Prees.

Jiang, Gengyu. (2006). Art Dialetic-Chinese Artic Wisdom Forms. Beijing : Higher Education Press.

Jung,Carl Gustav. (1997). Prototypes of Colletive Unconsciouseness, Prototypes and Collective Unconsciouseness from the Collected Works f C.G.Jung, Volume 9, the first fascicule. Beijing : Reform Press.

Liu Dachun. (2002). Scientific Activity Theory and Mutual Supplementation Theory. Guilin: Guangxi Normal University Press.

Pogson, F.L. (2004). Introducation à la Metaphysique. Beijing : Commercial Printing House.

Pogson, F.L. (2004). Time and Free Will. Beijing : Commercial Printing House.

\section{Notes}

Note 1. Movie, Mars Attacks, directed by Tim Burton and produced by Warner Brothers in 1996

Note 2. Please refer to the flower and birds paintings of Badashanren. The ture name of Badashanren is Zhu Long. He was from Nanchang, Jiangxi and lived at the end of Ming dynysty and at the begining of Qing dynysty. He is the nineth grandson of Zhu Quan, the Xian King of Ming Dynysty. He is one of the most famous painters of China and is the head of the "Four Monks", painting field at the begining of Qing dynysty. His works usally apply symblizing to express modds and are full of contumacy.

Note 3. Anselm Kiefer, a German famous painter of the new expressionism.

Note 4. Du Fu (Tang) Spring Perspective: The nations has fallen, the land endures: Spring tree and grasses flourish in the town. Troubled by the times - flowers bring tears; Dreading parting — birds startle the soul. With turmoil of battle three months on end. A letter from home is worth a fortune in gold. Scratching these white locks makes them thinner; As it is, they can barely hold a pin.

Note 5. Chinese ideom. The aftersound moves around the girder for three days' means that the music is so beautiful that in the three days after hearing, you will feel the rhythem is circuling in your room.

Note 6. Please refer to the works of the author The Art Branches of Altra-design Era and Decoration. Beijing, the first edition, 2007, p.130

Note 7 . The sensitivity refers to the acuity extent of vision observation. It is closely related to viional logic. The vision sensitivity difference of two persons is caused by inheritance and individual unconsciousness. The speed of feeling is due to the memory and wakening of visual remaining images, which is similar to the working ways of immune systems in biology. The immunnity means that a person hase more antibodies than commmon people. By this way, when attacked by the pathogeny, he or she could find antibodies quicker, make duplication timely and orginzie to defend his or her body, while for common people, their antibodies are obtained through injection or the remaining antibodies obtained from the previous disease. If this concept is used on the researches on visual sensitivity, the injection should be professional training and education while the remianing antibodies are the same as the memory and wakening of viisonal incomple images. Inherietn is inborn collective unconsciousness and is the sume of aesthetic evaluation formed in long term deposition and remaining images. 